\title{
Mutual influence of magnetic field decay and thermal evolution of rotational neutron stars
}

\author{
Xia Zhou ${ }^{1}$, Miao Kang ${ }^{2}$ and Na Wang ${ }^{1}$ \\ ${ }^{1}$ Xinjiang Astronomical Observatory, CAS, 150, Science 1-Street, Urumqi, 830011, China \\ Key Laboratory of Radio Astronomy, CAS, Urumqi, 830011, China. \\ email: zhouxia@xao.ac.cn, na.wang@xao.ac.cn \\ ${ }^{2}$ College of Physics and Electronics, Henan University, Kaifeng, Henan 475004, China \\ email: kangmiao07@gmail.com
}

\begin{abstract}
The effect of magnetic field decay on the chemical heating and thermal evolution of neutron stars is discussed. Our main goal is to study how chemical heating mechanisms and thermal evolution are changed by field decay and how magnetic field decay is modified by the thermal evolution. We show that the effect of chemical heating is suppressed by the star spin-down through decaying magnetic field at a later stage; magnetic field decay is delayed significantly relative to stars cooling without heating mechanisms; compared to typical chemical heating, the decay of the magnetic field can even cause the temperature to turn down at a later stage.
\end{abstract}

Keywords. stars: evolution, stars: magnetic fields, stars: neutron, stars: rotation, radiation mechanisms: thermal.

\section{Introduction}

Neutron stars (NSs) are detected as pulsars. Their regular pulsations in the radio, $\mathrm{X}$-ray, and/or optical bands are produced by a strong magnetic field being turned around at the stellar rotation period. It's widely accepted that a rapidly spinning neutron star loses its rotational energy by magnetic dipole radiation; thus the rotational evolution of isolated NSs are determined by the evolution of its magnetic field (Becker 2008, Glendenning 2000). NSs also are very hot at birth, with temperatures well above $10^{10} \mathrm{~K}$. This heat is radiated away mainly by neutrinos from the interior during the first million years or so (the neutrino cooling era) while later the emission of photons from the surface dominates the cooling of the star (the photon cooling era). This photon luminosity and its change with time depend on the properties of dense matter in the interior of NSs, its magnetic field and heating mechanisms (Becker 2008, Glendenning 2000). For example, the heating energy of chemical heating comes from the rotational energy which is converted into heating by storing rotational energy in terms of chemical energy (Reisenegger 1995, Fernández \& Reisenegger 2005). Observations of thermal radiation provide important information about the state of matter above and below nuclear density as well as for the magnetic field (e.g. Yakovlev et al. 2001, Zheng \& Zhou 2006, Zhou et al. 2007 and references therein). Goldreich \& Reisenegger (1992) discussed the processes which promote the dissipation of magnetic energy in NSs, while the decaying magnetic field of magnetar and how this decay affects the cooling of the stars is studied in Heyl \& Hernquist (1997, 1998) and Miralles et al. (1998).

In summary, the rotational, magnetic field and thermal evolution of NSs are coupled, and influence each other. Here we discuss the coupling evolution thermal evolution and magnetic field decay of rotating NSs, focusing especially on the effect of the decaying magnetic field on chemical heating mechanism. 


\section{The models}

Several physical mechanisms have been proposed for magnetic field decay in NSs: ohmic decay, ambipolar diffusion and Hall drift (Goldreich \& Reisenegger 1992). Depending on the strength of the magnetic field, each of these processes may dominate the evolution. A simple differential equation can be used to describe the dipole magnetic field decay (Goldreich \& Reisenegger 1992, Heyl \& Hernquist 1997, Heyl \& Hernquist 1998, Miralles et al. 1998):

$$
\begin{gathered}
t_{\text {ohmic }} \sim 2 \times 10^{11} \frac{L_{5}^{2}}{T_{8}^{2}}\left(\frac{n}{n_{0}}\right)^{3} \mathrm{yr}, t_{\text {ambip }}^{s} \sim 3 \times 10^{9} \frac{L_{5}^{2} T_{8}^{2}}{B_{12}^{2}} \mathrm{yr}, \\
t_{\text {ambip }}^{\text {irr }} \sim \frac{5 \times 10^{15}}{T_{8}^{6} B_{12}^{2}} \mathrm{yr}+t_{\text {ambip }}^{s}, t_{\text {Hall }} \sim 5 \times 10^{8} \frac{L_{5}^{2} T_{8}^{2}}{B_{12}}\left(\frac{n}{n_{0}}\right) \mathrm{yr} .
\end{gathered}
$$

Here we take $n=0.56 \mathrm{fm}^{-3}, \rho_{c}=1.2 \times 10^{15} \mathrm{~g} \mathrm{~cm}^{-3}, x_{e q}=0.07, R=10.4 \mathrm{~km}$; these are the typical values for a $1.4 M_{\odot}$ NS modeled with equation of state "AV14+UVII" (Wiringa et al. 1998). In that equation of state, the NS cools through modified URCA processes. With the given initial spin period, temperature and magnetic field, we solve the following equations numerically (Reisenegger 1995):

$$
\begin{gathered}
\frac{d \delta \mu}{d t}=-E_{x x}\left(\alpha n \frac{E_{n x}}{E_{x x}} \frac{\Omega \dot{\Omega}}{G \rho_{c}}+\frac{\Gamma}{n}\right), \frac{d B_{p}}{d t}=-B_{p}\left(\frac{1}{t_{\text {ohmic }}}+\frac{1}{t_{\text {ambip }}}+\frac{1}{t_{\text {Hall }}}\right), \\
\dot{E}_{B}=-\frac{1}{6} \frac{d B_{p}}{d t} B_{p} R^{3}, C_{v} \frac{d T}{d t}=\frac{\Gamma \delta \mu}{n}-\frac{\dot{E}_{\nu}}{n}-\dot{E}_{\gamma}+\frac{m_{n}}{M} \dot{E}_{B}, \frac{d \Omega}{d t}=-\frac{2 R^{6}}{3 c^{3} I} \Omega^{3}(t) B^{2}(t) .
\end{gathered}
$$

\section{Discussions and Conclusions}

The results are presented in Fig. 1. Our work shows that: the thermal evolution delays the decay of the magnetic field through heating effects; while the decaying magnetic field even makes the surface temperature become lower at photon cooling era through rotation and chemical evolution. The heating energy of chemical heating comes from the rotational energy which is converted into heating by storing rotational energy in terms of chemical energy. As follows from

$$
\frac{d \delta \mu}{d t}=-E_{x x}\left(\alpha n \frac{E_{n x}}{E_{x x}} \frac{\Omega \dot{\Omega}}{G \rho_{c}}+\frac{\Gamma}{n}\right),
$$

the evolution of $\delta \mu$ is closely connected with rotation evolution (through angular velocity directly or indirectly). In the photon cooling era $\delta \mu$ becomes small, since the spin-down of the star has been delayed by the decaying magnetic field. Meanwhile, the surface temperature of the star also becomes lower in the same era. These show that: the effect of chemical heating has been suppressed by the spin-down of the stars through decaying magnetic field at a later stage. When we discuss the cooling of NSs, we should take into account the coupling effect of decaying magnetic field and spin-down of NSs on the heating mechanisms.

We are aware of the fact that our model is simplified and can be improved in many respects. Firstly, a more realistic NS model should be considered more carefully. Secondly, a more elaborate model of the magnetic field evolution may be used, instead of a simple model formulated in Sect. 2, in order to take into account the variation of the magnetic field of the stars. Thirdly, the reaction rates in superfluid neutron stars are suppressed at low temperatures (Yakovlev et al. 2001) and superfluidity makes ambipolar diffusion inefficient (Glampedakis et al. 2011). These arguments suggest that the coupling evolution of a superfluid neutron star should be studied carefully in future work. In spite of some 

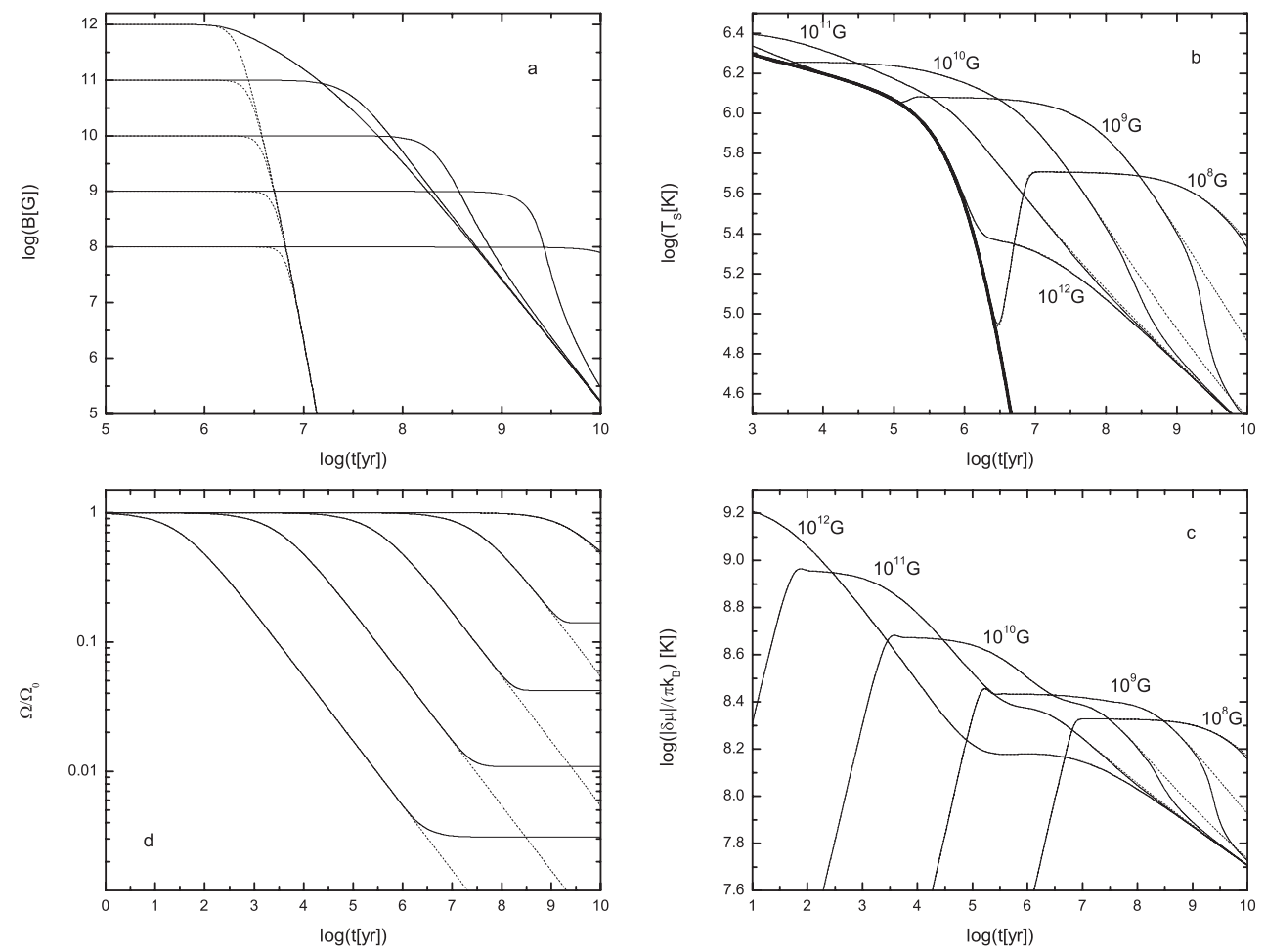

Figure 1. $a$ : Time evolution of the decaying magnetic field with chemical heating and magnetic field dissipation heating (solid lines). The curves for decaying fields which are cooling through the modified URCA processes without any heating effects (dotted lines); $b$ : Evolution of $\frac{\delta \mu}{\left(\pi k_{B}\right)}$ for different initial magnetic field; $c$ : Evolution of the surface temperature for different initial magnetic field. $d$ : Time evolution of normalized angular velocity with different initial magnetic fields. The solid lines are for decaying fields with chemical heating and magnetic field dissipation heating. The dotted lines are for the standard magnetic dipole braking model.

shortcomings, our simple model shows the qualitative importance of the coupling evolution of thermal, rotational and magnetic field of NSs, especially the chemical heating have been suppressed by the decaying magnetic field. Future investigations will consider a consistent evolution including the geometry of the magnetic field, the structure of NSs, and compared with the thermal emission observation data.

\section{References}

Becker, W. 2008. eds, Astrophys. Space Sci. Library Vol. 357, Neutron Stars and Pulsars, (Springer, New-York)

Fernández, R. \& Reisenegger, A., 2005. Astrophys. J. 625, 291.

Glampedakis, K., Jones, D. I., \& Samuelsson, L. 2011, Mon. Not. R. Astron. Soc., 413, 2021

Glendenning, N. K. 2000, Compact Stars: Nuclear Physics, Particle Physics, and General Relativity, (Springer, New-York)

Goldreich P., Reisenegger A. 1992, Astrophys. J., 395, 250

Heyl J. S. \& Hernquist L. 1997, Astrophys. J., 491, L95

Heyl J. S. \& Kulkarni S. R. 1998, Astrophys. J., 506, L61

Miralles J. A., Urpin V., \& Konenkov D. 1998, Astrophys. J., 503, 368

Reisenegger, A. 1995, Astrophys. J., 442, 749

Wiringa, R. B., Fiks, V., \& Fabrocini, A. 1988, Phys. Rev. C, 38, 1010

Yakovlev, D. G., Kaminker, A. D., Gnedin, O. Y., \& Haensel, P. 2001, Phys. Rep., 354, 1

Zheng, X. P. \& Zhou, X. 2006, Mon. Not. R. Astron. Soc., 371, 1659

Zhou, X., Wang, L. Z., \& Zhou, A. Z. 2007, Publ. Astron. Soc. Pac., 119, 1367 